\title{
Effects of Trypanosoma brucei tryptophanyl-tRNA synthetases silencing by RNA interference
}

\author{
Liliana Torcoroma García/*, Ney Ribeiro Leite, Juan D Alfonzo**, \\ Otavio Henrique Thiemann/*/+
}

Instituto de Física de São Carlos, Universidade de São Paulo, Caixa Postal 369, 13560-590 São Carlos, SP, Brasil *Departamento de

Genética e Evolução, Universidade Federal de São Carlos, São Carlos, SP, Brasil ** Department of Microbiology, Ohio State

Biochemistry Program and the Ohio State University RNA Group, The Ohio State University, Columbus, OH, US

The kinetoplast genetic code deviates from the universal code in that $90 \%$ of mitochondrial tryptophans are specified by UGA instead of UGG codons. A single nucleus-encoded tRNA ${ }^{\operatorname{Tr}}(C C A)$ is used by both nuclear and mitochondria genes, since all kinetoplast tRNAs are imported into the mitochondria from the cytoplasm. To allow decoding of the mitochondrial UGA codons as tryptophan, the $R_{R N A}{ }^{\operatorname{Tr} p}(C C A)$ anticodon is changed to UCA by an editing event. Two tryptophanyl tRNA synthetases (TrpRSs) have been identified in Trypanosoma brucei: TbTrpRS1 and TbTrpRS2 which localize to the cytoplasm and mitochondria respectively. We used inducible RNA interference (RNAi) to assess the role of TbTrpRSs. Our data validates previous observations of TrpRS as potential drug design targets and investigates the RNAi effect on the mitochondria of the parasite.

Key words: tryptophanyl tRNA synthetase - RNA interference - kinetoplastid - Trypanosoma brucei

Parasitic protozoa represent a considerable source of human diseases predominant in the tropical and subtropical regions of the globe. Of particular relevance are parasites belonging to the family Trypanosomatidae. Trypanosoma brucei and its subspecies are responsible for heavy socioeconomic losses in 36 sub-Saharan Africa countries (1.6 million DALYs) and lead to an estimated 48 thousand deaths per year. Therapy against African sleeping sickness has always been difficult, especially when the disease has reached an advanced stage with central nervous system involvement, as few effective drugs are available. For these reasons, the search and validation of new pharmacological targets against trypanosomiasis is of great concern.

Trypanosomatids are early diverging flagellates that differ from other eukaryotes in a number of features. They contain a remarkable single mitochondrion (the kinetoplast) harboring a large mass of circular DNA molecules concatenated in a unique arrangement, the kinetoplast DNA or kDNA (Lukes et al. 2002). The kinetoplast genetic code, like many other mitochondrial genomes and to the exception of plants, deviates from the universal code in that $90 \%$ of tryptophans are specified by UGA instead of UGG codons, although both codons are used in translation. A single nucleus-encoded tRNA $^{\operatorname{Trp}}(\mathrm{CCA})$ is used by both the nuclear and mitochon-

Financial support: CNPq (472570/2004-8), Fapesp (98/14138-2). LTG is a Capes doctorate fellow. NRL is a Fapesp fellow (02/ 01883-9). JDA is supported by a grant from the American Heart Association.

${ }^{+}$Corresponding author: thiemann @if.sc.usp.br

Received 27 April 2007

Accepted 1st August 2007 drial genes. Since all kinetoplast tRNAs are encoded in the nucleus and later imported into the mitochondria (Lima \& Simpson 1996), a C to U deamination event at position 34 of tRNA ${ }^{\operatorname{Tr}}(\mathrm{CCA})$ changes the anticodon from CCA to UCA allowing the decoding of UGA stop codons to Trp (Alfonzo et al. 1999).

The identification of two tryptophanyl tRNA synthetases (TrpRSs) proteins in T. brucei cells was reported (Charrière et al. 2006). TbTrpRS1 (cytoplasmic, accession no. XM821841) and TbTrpRS2 (mitochondrial, accession no. XM820510), where TbTrpRS2 is able of aminoacylate both the unedited tRNA ${ }^{\operatorname{Tr}}(\mathrm{CCA})$ and the edited tRNA ${ }^{\operatorname{Trp}}$ (UCA). We used inducible RNA interference (RNAi) in combination with biochemical techniques to assess the role of TbTrpRSs, thereby extending previous observations (Charrière et al. 2006) and validating the TrpRSs as potential drug design targets.

\section{MATERIALS AND METHODS}

The nucleic acid and amino acid sequences of both genes, TbTrpRS1 and 2, were used to search the T. brucei genomic databases, and the results showed no significant identity with other T. brucei genes as well as with their human and Escherichia coli homologues. A sequence identity of $57 \%$ is shared between TbTrpRS1 and 2 distributed along the entire sequence. Due to the low sequence identity, we chose the full length ORF sequences of TbTrpRS1 and 2 for RNAi analysis, based on the idea of increasing the specificity and efficiency of RNAi knockdown (LaCount et al. 2000). T. brucei 2913 procyclic cells were grown in SDM-79 supplemented with $10 \%$ fetal bovine serum in the presence of hygromycin $(50 \mu \mathrm{g} / \mathrm{ml})$ and $\mathrm{G} 418(15 \mu \mathrm{g} / \mathrm{ml})$ at $25-26^{\circ} \mathrm{C}$ without agitation (Wang et al. 2000). Each specific DNA for RNAi experiments was amplified by the polymerase chain reaction (PCR) from $T$. brucei 29-13 procyclic genomic DNA using the following gene-specific primers, containing the XhoI and HindIII (underlined) restric- 
tions sites: TbTrpRs1a 5'-CGCCTCGAGATGACTGAAGCCGTTGTAACACCGTGGTCT-3', TbTrpRs1b 5 ' - TCAAGCTTTCACTGGGTTGCCGCCTTCTTCGCTGGAC-3', TbTrpRs2a 5'-TACTCGAGATGCGCCGGGCTTCACATGTTTGCACGTC-3' and TbTrpRs2b 5'-CTAAGCTTCTATGCCATAATGTTGCGCACCTCCGTAAA-3'. The PCRs were carried out in a PTC-100 thermocycler (MJ Research Inc.) with 0.2 units of Taq DNA polymerase (Biotools) according to the manufacturer conditions. The samples were subjected to 3 min denaturation at $94^{\circ} \mathrm{C}$ followed by 20 cycles of denaturation at $94^{\circ} \mathrm{C}$ for $0.5 \mathrm{~min}$, annealing at $54^{\circ} \mathrm{C}$ for $0.5 \mathrm{~min}$, and extension at $72^{\circ} \mathrm{C}$ for $1.5 \mathrm{~min}$. A final step at $72^{\circ} \mathrm{C}$ for $10 \mathrm{~min}$ was added to maximize the full-length products. Each PCR fragment was cloned into the pCR2.1TOPO vector and sequenced prior to cloning into the XhoI/HindIII sites of the pZJM vector (Wirtz et al. 1999). These constructs were linearized by NotI cleavage and transfected into procyclic T. brucei $29-13$ cell line by electroporation as previously described (Wirtz et al. 1999, Wang et al. 2000). The transfected cells were cloned by the limiting dilution technique and selected under $2.5 \mu \mathrm{g} / \mathrm{ml}$ phleomycin until stable cell lines were obtained. To induce synthesis of the double stranded RNA (dsRNA), cells were incubated in medium containing tetracycline $(1.5 \mu \mathrm{g} / \mathrm{ml})$ for $24 \mathrm{~h}$ and transferred daily to fresh medium with tetracycline. Growth curves were obtained using cells fixed with $4 \%(\mathrm{v} / \mathrm{v})$ formaldhehyde in PBS and counted using a hemacytometer.

For cell morphology analysis, non-induced and tetinduced cultures were examined at different time intervals after RNAi induction by light microscopy. We used different staining techniques: (a) DAPI (4,6-diamidino2-phenylindole, Sigma) for nuclear and kinetoplast DNA. Cells were washed in PBS, and then fixed and stained with DAPI $(75 \mathrm{ng} / \mathrm{ml})$ for $1 \mathrm{~min}$ and analyzed by fluorescence microscopy at $350 \mathrm{~nm}$ of $\lambda$ excitation; (b) Mitotracker: the mitochondrion of living trypanosomes was visualized with the potential sensitive vital stain MitoTracker Red CMXRos ( $\lambda \mathrm{Em}$ 579nm and $\lambda \mathrm{Ex}$ $599 \mathrm{~nm}$ from molecular probe), a derivative of Xrosamine. Cells in $\log$ phase of growth were incubated with $100 \mathrm{nM}$ Mitotracker Red CMXRos at $28^{\circ} \mathrm{C}$ for 5 min in SMGHP, then, the cells were washed with fresh media and finally, incubated without dye for $20 \mathrm{~min}$; (c) $D A P I-R E D$ : fluorescence images of cells stained with $60 \mu \mathrm{M}$ DAPI and Mitotracker Red CMXRos $5 \mu \mathrm{M}$. For fixation, cells were washed with PBS and suspended in $4 \%$ freshly made paraformaldehyde in PBS. The percentage of morphologic anomalies (number nucleus, kinetoplasts, and form) was determined in 150 cells.

To determine the percentage of TbTrpRSs mRNA inhibition, total T. brucei RNA was isolated using Trizol (Life Technologies) after RNAi induction from the different cell lines at different time points. The TbTrpRS1 and TbTrpRS2 mRNA were estimated by semi-quantitative RT-PCR (Kit One Step RT-PCR from Qiagen) and by quantitative real-time PCR (QPCR), using the spliced leader (SLRNA) primer 5'-AACTAACGCTATATAAGTATCAGTTTCTGTACTTTATTG-3' (Campbell et al. 1984) and TbTrpRs $1 b$ and TbTrpRs2b primers. For the semi-quantitavite RT-PCR experiments $100 \mathrm{ng}$ of total RNA of each sample were added to a final reaction volume of $50 \mu \mathrm{l}$. The samples, after RT-PCR were analyzed in a $2 \%$ agarose gel. The bands were quantitated using the Kodak ds 1D digital science V.3.0.1 software. The analysis was performed comparing the intensity of the ethidium bromide stained bands of control (non-induced) and RNAi induced samples. QPCR was performed using the Absolute QPCR SYBR Green Mix (Abgene Advanced Biotecnologies Ltd.) as previously described (Yin et al. 2001) and performed in a Rotor Gene Real Time PCR (Corbett Research, Uniscience). The QPCR data obtained was analyzed using paired t-tests on the linear $2^{-\Delta \mathrm{Ct}}$ data set (Livak \& Schmittgen 2001). The RT-PCR and QPCR experiments of TbTrpRSs were performed. As a quantitation standard the T. brucei glycosomal glyceraldehydes 6-phosphate dehydrogenase (gGAPDH, $X 59955.1)$ gene was used with the primer pair TbGAPDHa 5'-CCTC GGGAATGAGATTGATGTCGTTGCTGT-3' and TbGAPDHb 5'-CACATACTCCACACCAAGCTTTCCC-3' in a standard PCR, as described above. Samples from induced, non-induced and non-transfected (control) cells were tested.

Oxygen uptake by $T$. brucei cells was measured with a biological oxygen monitor DM4 equipped (Digimed) with oxygen probe. The rate (ppm) was calculated by assuming the oxygen content of air-saturated water is 0.276 $\mathrm{mM}$ at $28^{\circ} \mathrm{C}$ (according to manufacturer instructions). The cell concentration used was $1 * 10 \mathrm{E} 6$ cells $/ \mathrm{ml}$.

\section{RESULTS}

RNAi expression against TbTrpRS1 resulted in severe growth inhibition $4 \mathrm{~h}$ after induction (reduction of $84 \%$ relative to control cells) with cell death after $24 \mathrm{~h}$ of induction. TbTrpRS2 knockdown after $6 \mathrm{~h}$ of tetracycline induction produced a $79 \%$ growth inhibition and complete cell death after $72 \mathrm{~h}$ of induction. Both cell lines exhibited eventual complete arrest of growth, which was more quickly achieved in post-induced TbTrpRS1 cultures $(24 \mathrm{~h})$ than in TbTrpRS2 $(72 \mathrm{~h})$, as shown in Figs 1,2. These cultures showed no recovery after release from tetracycline induction, confirming the lethal and irreversible effect of the gene knockdowns (data not shown).

Expression of TbTrpRS1 dsRNA led to total degradation of the specific mRNA, which was undetectable by semi-quantitative RT-PCR. QPCR analysis revealed that transcript levels were reduced more than 16 fold in cells after $24 \mathrm{~h}$ of TbTrpRS1 dsRNA induction (Fig. 2). Expression of TbTrpRS2 dsRNA also resulted in an efficient degradation of the cognate mRNA resulting in an approximately 3 -fold decrease after $48 \mathrm{~h}$ of induction and a complete silencing at $72 \mathrm{~h}$ of induction, after which TbTrpRS2 mRNA amounts were not detectable by either semi-quantitative RT-PCR or QPCR (levels < 13 folds) (Fig. 2). These data confirmed the efficiency of silencing of expression of the TbTrpRSs by RNAi. Transcript levels were not significantly changed in the non-induced TbTrpRS2 controls or in the wild type T. brucei 29-13 cells $(P=0.62)$. An unrelated transcript for $T$. brucei gGAPDH remained unaffected in the samples analyzed. Furthermore, silencing of one TbTrpRS gene did not in- 
fluence the expression of the other (data not shown). The non-induced T. brucei cells transfected with the TbTrpRS1 RNAi construct exhibited a small reduction ( 0.039 folds; $P=0.06$ ) of the TbTrpRS $1 \mathrm{mRNA}$ as compared to the non-transfected $T$. brucei $29-13$ cells. This phenomenon can be explained by a leakage in the T7 dualpromoter system, which has been previously observed (Durand-Dubief et al. 2003).
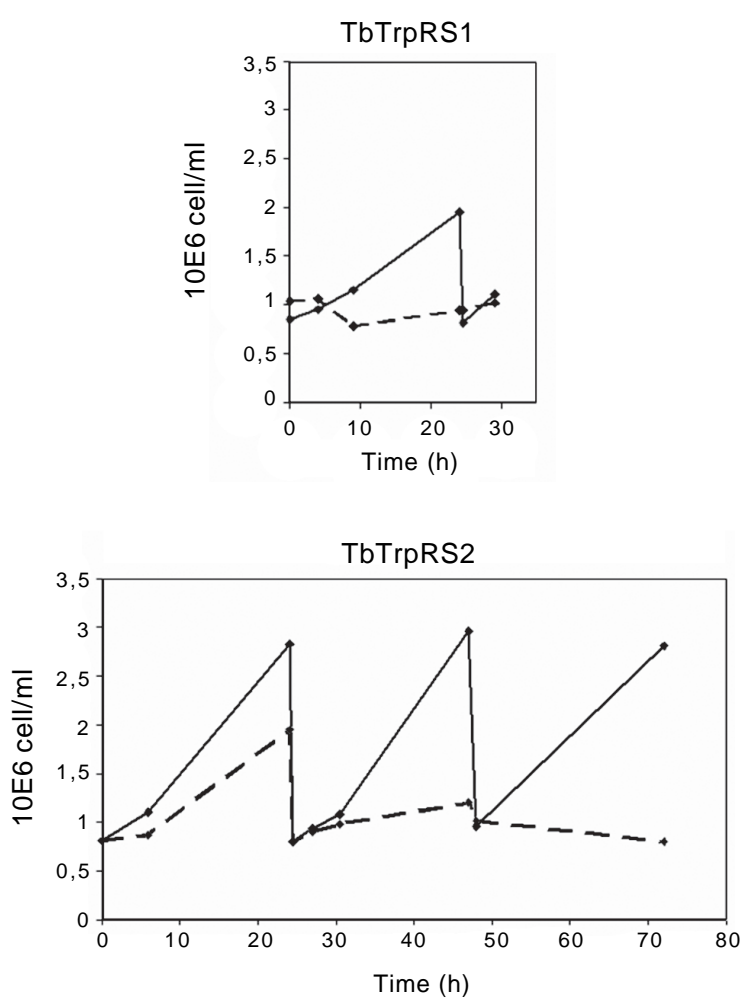

Fig. 1: growth of tetracyclin induced (dotted line) and non-induced (solid line) TbTrpRS1 and 2 RNAi strains of Trypanosoma brucei 29-13 cells.

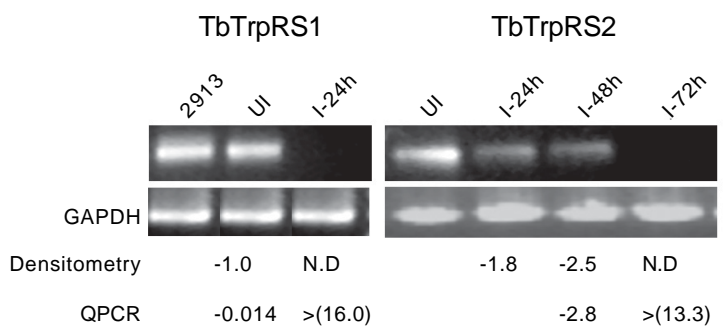

Fig. 2: reverse-transcription-polymerase chain reaction (RT-PCR) and quantitative real-time PCR (QPCR) of Trypanosoma brucei citoplasmatic tryptophanyl tRNA synthetase (TbTrpRS1) and 2 mRNA. 2913: nontransfected cells. UI: TbTrpRS1 non-induced cells and I-24 h, I-48 h and $\mathrm{I}-72 \mathrm{~h}$ cells induced after 24,48 , and $72 \mathrm{~h}$, respectively. GAPDH gene was used as a control and is shown for comparison. Quantification was performed by both, band densitometry (Densitometry lane) from semi-quantitative RT-PCR gel bands shown in the figure and by QPCR (data not shown).
To analyze the cell morphology, non-induced and tetracycline induced cultures were examined. The phenotype of the control (non-induced) cells appeared normal (Figs 3A, 4A). The distribution of cell morphologies of induced and non-induced knockdowns is presented in the Table. The cells were classified in two categories according to the number of kinetoplasts $(\mathrm{K})$ and nuclei $(\mathrm{N})$ per cell. "Normal" constituted cells with $1 \mathrm{~K} 1 \mathrm{~N}$ or with $2 \mathrm{~K} 1 \mathrm{~N} / 2 \mathrm{~K} 2 \mathrm{~N}$, representing different stages of cell division. Cells were classified as "abnormal" when containing a different number of K or N. TbTrpRS1 knockdown cells also presented a round phenotype and were classified as 'spheres'. In the control cultures, the population mainly (90-94\%) constituted of $1 \mathrm{~K} 1 \mathrm{~N}$ cells and a minority of dividing cells $(2 \mathrm{~K} 1 \mathrm{~N}$ or $2 \mathrm{~K} 2 \mathrm{~N})$ as shown in Fig. 4A and in the Table.

In the TbTrpRS1 RNAi cells, morphological abnormalities can be seen after $9 \mathrm{~h}$ of tetracycline induction. At $24 \mathrm{~h}$ after tetracycline induction the majority of cells $(41 \%)$ increased in size, and contained numerous nuclei, kinetoplast, and several flagella, suggesting inhibition of cytokinesis. A significant percentage (33\%) presented the 'sphere' phenotype lacking flagella (Table, Fig. 3B). The TbTrpRS2 knockdown leads to a wide range of morphologic abnormalities. These phenotypes included cells with partially detached flagella (two or more flagella) (Fig. 3B), cells with incomplete cell separations (Fig. 3C), mini-trypanosomes that lack nuclei (Fig. 4D) and cells with defects in cytokinesis (multinucleated; Fig. $4 \mathrm{E})$. At $24 \mathrm{~h}$ after tetracycline induction, a high proportion of the cells $(36 \%)$ presented abnormalities in their morphology (Figs 3B, 3C, 4B) compared to untreated cells, in which the percentage of abnormal cells was no more than $10 \%$ (Table). The depletion of TbTrpRS2 resulted in the accumulation of mini-trypanosomes that lack nuclei, suggesting an interruption of mitosis without inhibiting kinetoplast segregation, which led to cytokinesis and cell division and generated enucleated daughter cells (Ploubidou et al. 1999). In Figs 3 and 4 are shown some representative types of cells found in the knockdown of TbTrpRS1 and 2.

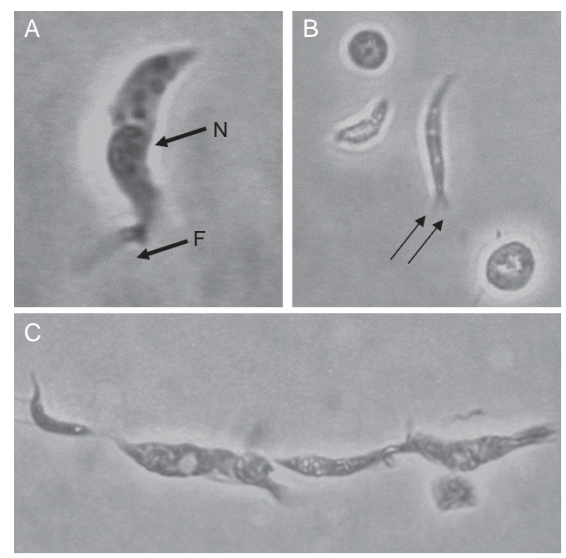

Fig. 3: phase contrast images from Trypanosoma brucei cells. A: noninduced TbTrpRS1 cells. Arrows indicate N: nucleus; F: flagella; B: TbTrpRS1 cells $24 \mathrm{~h}$ after tetracycline induction. Arrows indicate cell with several detached flagella; $\mathrm{C}$ : TbTrpRS 2 cells $48 \mathrm{~h}$ after RNAi induction. 
TABLE

Cell morphology of Trypanosoma brucei cells after RNAi induction

\begin{tabular}{|c|c|c|c|c|c|c|c|c|c|c|}
\hline \multirow[b]{2}{*}{ Cells } & \multicolumn{3}{|c|}{ Normal } & \multicolumn{7}{|c|}{ Abnormal } \\
\hline & $\underset{\%}{1 \mathrm{~K} 1 \mathrm{~N}}$ & $\underset{\%}{2 \mathrm{~K} 1 \mathrm{~N}}$ & $\underset{\%}{2 \mathrm{~K} 2 \mathrm{~N}}$ & $\begin{array}{c}1 \mathrm{~K} 0 \mathrm{~N} \\
\%\end{array}$ & $\underset{\%}{2 \mathrm{~K} 0 \mathrm{~N}}$ & $\underset{\%}{0 \mathrm{~K} 2 \mathrm{~N}}$ & $\underset{\%}{1 \mathrm{~K} 2 \mathrm{~N}}$ & $\underset{\%}{0 \mathrm{~K} 0 \mathrm{~N}}$ & $\underset{\%}{1 \mathrm{~K} 3 \mathrm{~N}}$ & $\begin{array}{c}\text { Sphere } \\
\%\end{array}$ \\
\hline $\begin{array}{l}\text { T.b. } 29-13 \text { control } \\
\text { TbTrpRS } 1\end{array}$ & 83 & 11 & 3 & 3 & & & & & & \\
\hline Non-induced & 79 & 11 & 3 & 3 & & 2 & 2 & & & \\
\hline Induced $24 \mathrm{~h}$ & 9 & 2 & 3 & 3 & & 3 & 3 & 3 & 41 & 33 \\
\hline TbTrpRS2 & & & & & & & & & & \\
\hline Non-induced & 78 & 12 & 2 & 4 & & 2 & 2 & & & \\
\hline Induced $24 \mathrm{~h}$ & 51 & 13 & 5 & 9 & 5 & 6 & 2 & 8 & 1 & \\
\hline Induced $48 \mathrm{~h}$ & 48 & 8 & 6 & 16 & 4 & 2 & 6 & 8 & 2 & \\
\hline Induced $72 \mathrm{~h}$ & 21 & 4 & 19 & 43 & 4 & 4 & 2 & 1 & 2 & \\
\hline
\end{tabular}

K: kinetoplast; N: nucleus; T.b. 29-13 control: wild type T. brucei 29-13 cells; TbTrpRS1: TbTrpRS1 transfected T. brucei 29-13 cells; TbTrpRS2: TbTrpRS2 transfected T. brucei 29-13 cells.

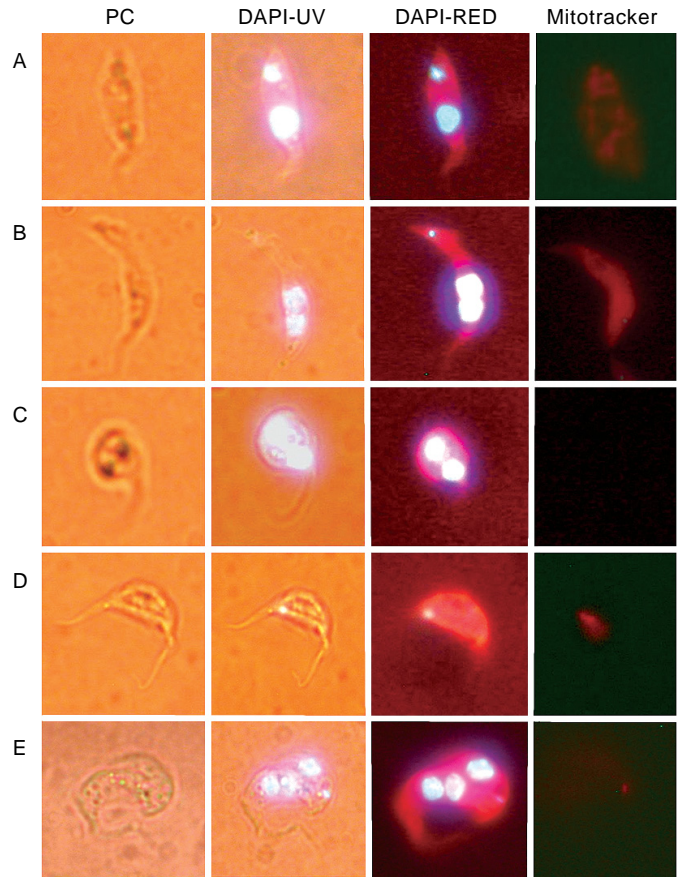

Fig. 4: cell morphology, kinetoplast, and nuclear DNA of Trypanosoma brucei mitochondrial tryptophanyl tRNA synthetase (TbTrpRS2) strain after different periods of incubation with tetracycline. PC: phase contrast images. DAPI-UV: DAPI stained viewed using both, visible and UV lights. DAPI-RED: fluorescence images of cells stained with Mitotracker Red CMXRos and DAPI stained observed in the DAPI channel; Mitotracker: fluorescence images of cells stained with the potential sensitive vital stain Mitotracker Red CMXRos in live cells; A: non-induced TbTrpRS2; B: $24 \mathrm{~h}$ after RNAi induction; C: $48 \mathrm{~h}$ after RNAi induction; D: example of Zooid morphology that appeared after $48 \mathrm{~h}$ of RNAi induction; E: $72 \mathrm{~h}$ after RNAi induction.

We used the mitochondria-specific stain, Mitotracker (molecular probes), in order to assay functional changes occurring during TbTrpRS2 RNAi induction. A typical reticular pattern is visible throughout the non-induced cells, representing the stained mitochondria, and this patter is significantly altered after TbTrpRS2 RNAi induction. Induced cells show a decrease in the mitochondria stain, in particular after $48 \mathrm{~h}$ of induction (Fig. 4C), indicating dramatic changes of metabolic function. To corroborate these results, the respiration rate or demand of oxygen (DO) was determined using a biological oxygen monitor DM4 (Digimed). The uninduced cells exhibit non-significant changes in their respiration rate compared with the control $(P=0.789)$. The respiration rate (ppm) was significantly decreased (64\%) at $48 \mathrm{~h}$ post-induction (Fig. 5). This result is consistent with the three-fold decrease in TbTrpRS2 mRNA (Fig. 2). After $72 \mathrm{~h}$ the DO from these cells was undetectable. The quantitative mRNA analysis from TbTrpRS2 RNAi induced samples suggested that the decrease in the DO and the reduction of the mitochondrial potential occurred after the mRNA depletion (Figs 5, 6). According to these results, we can conclude that slight perturbations in the expression of TbTrpRS2 can trigger significant and immediate alterations in the mitochondrial function and redox potential. In addition, at $72 \mathrm{~h}$ post-induction, the complete degradation of specific mRNA in the knockdown cells was compatible with the complete lost of respiration activity and mitochondrial membrane potential (Figs 5, 6). Furthermore, the inhibition of TbTrpRS2 synthesis could decrease the levels of mitochondrial translation, with a decline of mitochondrial redox potential, respiration capability and finally triggering cell death. The TbTrpRS1 RNAi induced deleterious phenotype (Figs 1,2) prevented an investigation of its role in mitochondria function, although such a role seems improbable.

\section{DISCUSSION}

The results we report are consistent with previous observations (Charrière et al. 2006) describing the existence of two TrpRSs genes in T. brucei cells and that knockdown of TbTrpRSs by RNAi effectively inhibits the replication of T. brucei procyclic forms in culture. In our experiments we extended those observations by the use of full length TbTrpRS1 and 2 genes in the RNAi 


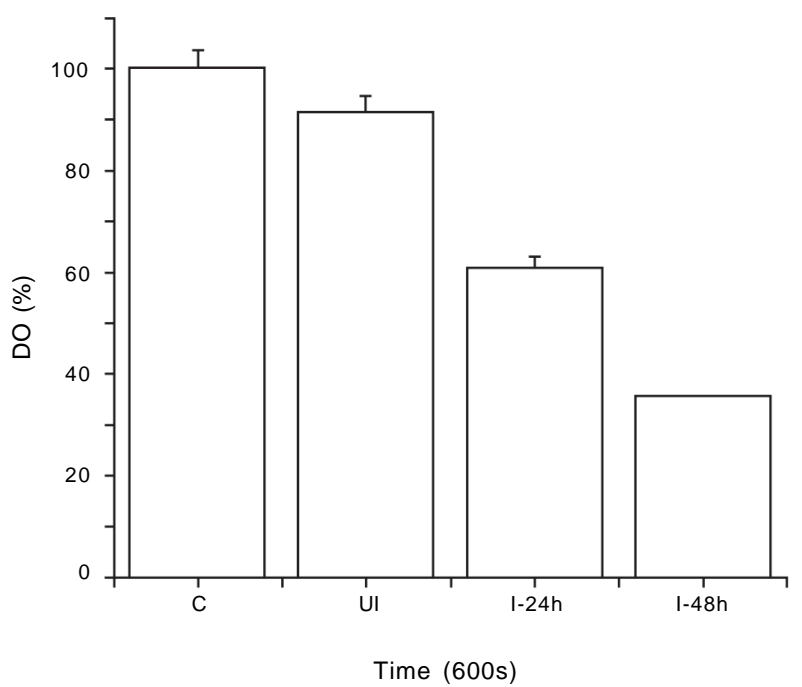

Fig. 5: assessment of respiratory function, oxygen demand (DO) of Trypanosoma brucei mitochondrial tryptophanyl tRNA synthetase (TbTrpRS2). C: T. brucei 29-13 control cells; UI: non-induced TbTrpRS2; $\mathrm{I}-24 \mathrm{~h}$ and I-48 $\mathrm{h}$ : TbTrpRS2 $24 \mathrm{~h}$ and $48 \mathrm{~h}$ after induction respectively. At $72 \mathrm{~h} \mathrm{DO}$ was non-detectable in this assay.

constructs. This contributed to a more pronounced sequence-specific knockdown, leading in a clearer physiological effect. Our results show that the outcome of RNA interference on TbTrpRS2 is a rapid decrease of mitochondrial activity, leading to a rapid cell death. The rapid rate which mutant phenotypes develop after expressing TbTrpRSs dsRNA indicate that these proteins have short half-lives since those enzymes are not abundant in the cells (data not shown). Our results confirm the central role that TbTrpRS1 and 2 play in cell viability, validating these proteins as targets for inhibitor development. In particular TbTrpRS2 is of interest due to its low sequence identity to other TbTrpRSs genes, especially that of humans, and for its role in blocking mitochondrial activity and resulting in cell death.

\section{ACKNOWLEDGEMENTS}

To the members of the Protein Crystallography and Structural Biology Group (IFSC-USP) for the helpful discussions in the course of this work. To Prof. Dr David Campbell Laboratory for providing pZJM vector and T. brucei 29-13 procyclic cells (Microbiology Immunology Molecular Genetics, University of California), Prof. Dr Heloisa Selistre de Araujo (LBBM, Federal University of São Carlos) for the QPCR support and Prof. Dr Luiz Antonio Daniel (São Carlos Engineering School, University of São Paulo) for support in the DO measurements.

\section{REFERENCES}

Alfonzo JD, Blanc V, Estevez AM, Rubio MA, Simpson L 1999. $\mathrm{C}$ to $\mathrm{U}$ editing of the anticodon of imported mitochondrial tRNATrp allows decoding of the UGA stop codon in Leishmania tarentolae. EMBO J 18: 7056-7062.

Campbell DA, Thornton DA, Boothroyd JC 1984. Apparent discon-

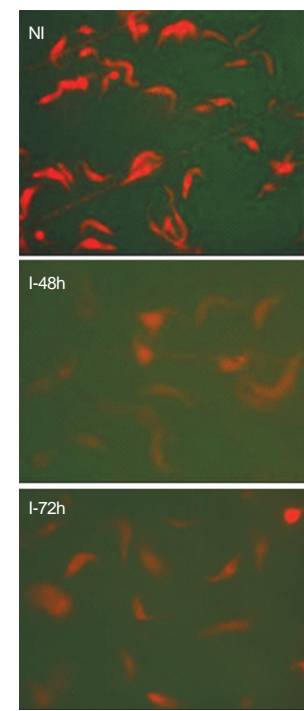

Fig. 6: fluorescence images of cells stained with Mitotracker Red CMXRos showing the accumulation of the redox potential sensitive dye by procyclic Trypanosoma brucei mitochondrial tryptophanyl tRNA synthetase (TbTrpRS2). NI: non-induced TbTrpRS2 cells; I-48 h and I-72 h: TbTrpRS2 cells $48 \mathrm{~h}$ and $72 \mathrm{~h}$ after tetracycline induction, respectively.

tinuous transcription of Trypanosoma brucei variant surface antigen genes. Nature 311: 350-355.

Charrière F, Helgadottir S, Horn, EK, Söll D, Schneider A 2006. Dual targeting of a single tRNATrp requires two different tryptophanyl-tRNA synthetases in Trypanosoma brucei. PNAS 103: 6847-6852.

Durand-Dubief M, Kohl L, Bastin P 2003. Effieciency and specificity of RNA interference generated by intra- and intermolecular double stranded RNA in Trypanosoma brucei. Mol Bioch Parasitol 129: 11-21.

Kim S, Lee SW, Choi EC, Choi SY 2003. Aminoacyl-tRNA synthetases and their inhibitors as a novel family of antibiotics. Appl Microbiol Biotechnol 61: 278-288.

LaCount DJ, Bruse S, Hill KL, Donelson JE 2000. Doublestranded RNA interference in Trypanosoma brucei using head-to-head promoters. Mol Bioch Parasitol 111: 67-76.

Lima BD, Simpson L 1996. Sequence-dependent in vivo importation of tRNA into the mitochondrion of Leishmania tarentolae. RNA 2: 429-440.

Livak KJ, Schmittgen TD 2001. Analysis of relative gene expression data using real-time quantitative PCR and the 2Delta Delta CT Method. Methods 25: 402-408.

Lukes J, Guilbride DL, Votýpka J, Zíková A, Benne R, Englund PT 2002. Kinetoplast DNA network: evolution of an improbable structure. Eukaryotic Cell 1: 495-502.

Ploubidou A, Robinson DR, Docherty RC, Ogbadoyi EO, Gull K 1999. Evidence for novel cell cycle checkpoints in trypanosomes: kinetoplast segregation and cytokinesis in the absence of mitosis. J Cell Sci 112: 4641-4650.

Wang Z, Morris JC, Drew ME, Englund PT 2000. Interference of Trypanosoma brucei gene expression by RNA interfer- 
ence using integratable vector with opposing T7 promoters. J Biol Chem 275: 40174-9.

Wirtz E, Leal S, Ochatt C, Cross GA 1999. A tightly regulated inducible expression system for conditional gene knockouts and dominant-negative genetics in Trypanosoma brucei. Mol Biochem Parasitol 99: 89-101.
Yin JL, Shackel NA, Zekry A, McGuinness PH, Richards C, van der Putten K, McCaughan GW, Eris JM, Bidhop GA 2001. Real-time reverse transcriptase-polymerase chain Reaction RT-PCR for measurement of cytokine and growth factor mRNA expression with fluorogenic probes or SYBR Green I. Immunol Cell Biol 79: 213-221. 